\title{
LOS LEPROSOS EN EL PORTUGAL DE LOS SIGLOS XIV Y XV: CONTRIBUCIÓN PARA UNA «HISTORIA DE LOS ASISTIDOS»
}

\author{
Rita Luis Sampaio Da Nóvoa ${ }^{1}$
}

\begin{abstract}
RESUMEN
La historiografía dedicada a la lepra, a los leprosos y a las leproserías en la época medieval ha desarrollado en las últimas décadas interesantes y innovadoras perspectivas de análisis. El trabajo enseguida presentado intenta explorar algunas de esas perspectivas aplicadas al caso portugués en los siglos XIV y XV. Entre varios aspectos, el principal objetivo es llamar la atención para la noción de leproso y para los diferentes factores que deben ser tenidos en consideración cuando el historiador piensa lo individuo enfermo. En realidad, la diversidad y complejidad de las situaciones verificadas exige no un abordaje simplista y general (leproso igual a excluido) pero una mirada que no deje de lado los aspectos que definen y caracterizan el leproso mas allá de la lepra.
\end{abstract}

Palabras clave: Siglos XIV-XV, Portugal, Lepra, Leprosos, Leproserías, «Historia de los asistidos».

\begin{abstract}
In the last decades the historiography dedicated to the study of leprosy, lepers and leperhouses in the Middle Ages has developed several innovative and interesting perspectives. The following article will explore some of them in relation to the portuguese scenario in the $14^{\text {th }}$ and $15^{\text {th }}$ centuries. Among other aspects, the main objective is to draw attention to the notion of leper and to the different factors that should be taken into consideration when the historian observes the sick individual. In fact, the diversity and complexity of the documented situations demands not a simplistic and generalist approach (leper as excluded) but a survey that doesn't exclude the characteristics that define the leper besides the leprosy itself.
\end{abstract}

Keywords: $14^{\text {th }}-15^{\text {th }}$ centuries, Portugal, Leprosy, Lepers, Leper-houses, «History of the Assisted».

Fecha de recepción: 25 octubre 2009. Fecha de aceptación: 22 diciembre 2009.

1 Faculdade de Ciências Sociais e Humanas. Universidade Nova de Lisboa. Portugal. Email: ritalsnovoa@yahoo.com. 
En las dos últimas décadas se ha asistido a un susceptible impulso revisionista en el área de la historiografía dedicada a la lepra, a los leprosos y a las leproserías en la época medieval. Entre otros trabajos, la monumental obra del medievalista francés FrançoisOlivier Touati - Maladie et société au Moyen Âge $e^{2}$ es una de las que mejor traduce las nuevas corrientes susceptibles de ser estudiadas, esas corrientes que no sólo contemplan perspectivas «clásicas» (como la «historia de la medicina» o la «historia de las instituciones») también enfoques innovadores (de los que la «historia de las actitudes» y la «historia de los asistidos» son ejemplo).

En rasgos generales, una de las principales presuposiciones de esta «nueva historia» se centra en el carácter complejo y, a veces, ambiguo de las realidades sobre las que se inclina. En otras palabras, se cuestiona la utilidad metodológica de las ecuaciones como «lepra igual a contagio», «leprosos igual a pobres y excluidos»o «leproserías igual a segregación social» ${ }^{3}$, que tanto les gusta a los estudiosos de finales del siglo XIX e inicios de la centuria siguiente. Añadiendo algo más, se ha declarado la guerra a la carga simbólica asociada con aquella enfermedad «medieval» — cuya única adversaria en la lucha por el título sería la peste-, en el mismo momento en que se apelaba a la deconstrucción de los «residuos tóxicos» 4 en ella contenidos.

En el mismo sentido, se ha asistido a una acentuación de la preocupación de la dimensión social de la enfermedad considerada indisociable de su componente biológica. Entonces se denunciaron las trampas historiográficas representadas por aquello a lo que se ha apellidado «ontologismo nosológico» y «diagnóstico retrospectivo» ${ }^{5}$ en una tentativa de garantizar la historización de la enfermedad. «Enfermedad de Hansen» y «el mal de San Lázaro» han dejado, por tanto, de ser entendidos como sinónimos, tomando consciencia del considerable número de peligros que ocasiona la busca de líneas de continuidad entre las respuestas sociales desarmadas por todos lados.

El trabajo presentado ahora es heredero de estas líneas historiográficas y, como tal, procurará avanzar en el sentido de aquello a lo que se le ha llamado «historia de los asistidos». Así, me propongo analizar las fuentes medievales que contienen referencias sobre la lepra, los leprosos y las leproserías y buscar en ellas la perspectiva del individuo enfermo con respeto a las opciones y condiciones que la sociedad le concede. El epicentro del análisis será, por tanto, el enfermo y no la enfermedad o la institución.

Por esa razón, aquí no sólo se tratará la percepción que la medievalidad da de la enfermedad (desde el punto de vista médico o religioso) o del conjunto de actitudes detectado en el seno de los grupos sanos en relación a los enfermos. Se van a tratar de conjugar

2 TOUATI, François-Olivier: Maladie et société au Moyen Âge. La lèpre, les lépreux et les léproseries dans la province ecclésiastique de Sens jusqu'au milieu du XIVe siècle, Paris, De Boeck Université, 1998.

3 TOUATI, François-Olivier: «Contagion and leprosy: myth, ideas and evolution in medieval minds and societies», in Contagion: perspectives from Pre-modern society, Ashgate, 2000, p. 181.

4 La expresión es de GEARY, Patrick: O Mito das Nações. A invenção do nacionalismo, Lisboa, Gradiva, 2008, p. 42.

5 Vd. ARRIZABALAGA, Jon: «La identificación de las causas de muerte en la Europa pre-industrial: algunas consideraciones historiográficas», in Boletin de la Asociacion de Demografia Historica, vol. 11, n. ${ }^{\circ} 3$, 1993, pp. 23-47 e CUNNINGHAM, Andrew: «Identifying disease in the past: cutting the Gordian knot», in Asclepio, vol. LIV-1, 2002, pp. 13-34. 
estos aspectos - estas respuestas sociales del otro - con los elementos identificados como intrínsecos al propio enfermo.

Así, voy a empezar por evaluar la noción de leproso y las perspectivas historiográficas que el medievalista puede adoptar en lo referido a la interpretación de las fuentes. Enseguida, pasaré al análisis de los casos conocidos en el Portugal de los siglos XIV y XV. La documentación utilizada en este ejercicio se refiere sobretodo a las grandes ciudades del reino que tenían a su disposición al menos una leprosería. Entre las tipologías documentales contempladas no sólo se cuenta con los estatutos de dichas leproserías, también con legaciones testamentarias, documentación enfitéutica, documentación regia y posturas municipales. De aquí resulta que el escenario, que ahora visitaremos, es predominantemente urbano, laico y marcado por percepciones y diferentes actitudes frente a la lepra con respeto a siglos anteriores. Entonces como caracterizamos la noción de leproso?

\section{LA NOCIÓN DE LEPROSO}

Algunas líneas antes he hecho referencia a la conocida caracterización del leproso como pobre y excluido, noción que ha sido fuertemente criticada y progresivamente desmontada por diversos autores. La base de sus ataques está, por un lado, en el regreso al origen de la construcción del «mito» y en la toma de consciencia de las presuposiciones médicas y de los propósitos políticos que han estado en su base ${ }^{6}$. Por otro lado, la lectura atenta de los registros medievales no ha permitido la sobrevivencia de un arquetipo fijo de pobreza y exclusión social, revelando múltiples y diversas realidades, resistentes a las tentativas de generalización.

A mi entender, la diversidad con la que el historiador se coloca puede ser explicada con la siguiente premisa: la enfermedad no anula al individuo. Esto quiere decir que los factores que definen al hombre son a los ojos de la sociedad (y que preexisten al factor enfermedad) que no desaparecen cuando surge la enfermedad. Así, al término leproso deben ser asociados una serie de aspectos dentro de la que la enfermedad sólo es uno y no necesariamente el más importante. Me refiero, a título de ejemplo, a los locales de nacimiento y residencia del individuo, a la familia en la que ha nacido, a su estatuto social, a sus posesiones económicas o al grado de proximidad con la curia regia.

Estos y otros elementos — que son independientes del «dolor de San Lázaro»— van a desempeñar un papel importantísimo para comprender la situación en la que determinado enfermo se encuentra. El historiador no se debe contentar, por tanto, con una definición demasiado simple, ni demasiado rápida del término de leproso. Veamos el porqué.

\section{LÁZAROS DOMÉSTICOS, LÁZAROS ERRANTES POR EL MUNDO Y RESI- DENTES EN LAS LEPROSERÍAS}

Teniendo en cuenta los ejemplos conocidos parece seguro afirmar que, a partir del momento en que se manifiestan las señales exteriores de la enfermedad, el leproso se

6 RAWCLIFFE, Carole: Leprosy in Medieval England, Woodbridge, The Boydell Press, 2006, pp. 1343. 
ve forzado a revisar total o parcialmente sus papeles sociales y/o los trámites de su vida cotidiana. Este proceso está directamente relacionado con el carácter específico de la enfermedad, que progresivamente han construido en torno a las nociones de «peligro»e de «contagio». Como ha demostrado F.-O. Touati ${ }^{7}$, la definición de lepra como enfermedad contagiosa no es transversal a todos los siglos que componen el período medieval, surgiendo sólo a finales de la centuria del Doscientos, hecho que también se verifica para el reino portugués.

En este sentido, la respuesta social o, si queremos, el mecanismo profiláctico primeramente activado en las grandes ciudades parece ser la expulsión del enfermo para el exterior del núcleo urbano. Así se garantizaba aquello que Foucault ha llamado «purificación del espacio urbano» ${ }^{8}$. Las determinaciones redactadas por la edilidad de la ciudad de O Porto en 1401 son ejemplo de esto al estipular las penas que los lázaros sufrirían en el caso de entrar en el corazón de la ciudad, esas penas que inclusivamente prevenían represalias físicas 9 .

Mediante la amenaza de expulsión, los enfermos se encuentran con, al menos, tres escenarios posibles en contexto urbano: la reclusión doméstica, la errancia «por el mundo» o el ingreso en la leprosería. No se puede excluir la existencia de otros destinos (relacionados, por ejemplo, con determinadas formas de organización religiosa), pero parece ser este el abanico de opciones más común.

En primer lugar, observaremos el ejemplo de la reclusión doméstica. Evitando la expulsión, los «lázaros domésticos» son aquellos que obtienen autorización para permanecer en sus residencias, comprometiéndose a eso y a evitar cualquier contacto con personas sanas fuera del espacio domiciliario. Las únicas autorizaciones de este género conocidas en el reino portugués han sido emitidas por el Monarca, anulando disposiciones previstas por las autoridades locales. Aún desconociendo si existía la posibilidad de obtener tal autorización por intermedio de otra entidad que no fuese el Rey, el hecho es que esta opción parece estar disponible sólo para un número limitado de enfermos.

Esto porque, además de presuponer la capacidad de apelar a instancias superiores, requería también disponibilidad para el sustento del leproso, impedido de abandonar su domicilio. Tal impedimento significaba que el enfermo doméstico tendría a su disposición los recursos necesarios para su supervivencia y bases de apoyo familiares $-o$, en principio, no institucionalizadas - que le permitían compensar la reclusión. Es decir, ¿la reclusión doméstica presuponía un estatuto social y/o económico elevado?

Los testimonios portugueses parecen indicar que sí. En 1460, D. Afonso V autorizó a que no abandonase su residencia a la mujer leprosa del escudero de un capitán mayor de Tánger, mientras le impedía la justicia local de Évora permanecer en su casa ${ }^{10}$. Ya en el

7 TOUATI, François-Olivier: «Contagion and leprosy: myth, ideas and evolution in medieval minds and societies», in Contagion: perspectives from Pre-modern society, Ashgate, 2000, pp. 179-201.

8 FOUCAULT, Michel: «O nascimento da medicina social», in A microfísica do poder, Brasil, Edições Graal, 2008, p. 88.

9 Vereaçoens (1401-1449), ed. PEREIRA, J.A. Pinto, Porto, Câmara Municipal do Porto, 1980, p. 45.

10 IAN/TT, Chancelaria de D. Afonso V, liv. 22, fl. 64. 
siglo XVI, la misma autorización se extiende a dos mujeres «honradas» $\mathrm{y}$ «con bienes», parientes de un mercader que servía a la Reina ${ }^{11}$.

Estos dos ejemplos son sintomáticos de la proximidad (directa o indirecta) de los requerientes con respeto a los miembros de la familia real y revelan también un estatuto socioeconómico relativamente elevado. En la misma línea, el primer caso denota la importancia de las referidas bases de apoyo familiares, porque es el esposo de la enferma el que dirige el pedido al Rey, afirmando específicamente que «la quería tener en su casa». El segundo ejemplo se refiere a la disponibilidad económica para soportar los costes de la reclusión doméstica, esa disponibilidad que anulaba la necesidad de tener otros apoyos institucionales o caritativos.

Incluso admitiendo la existencia de otras situaciones de reclusión doméstica diferentes a estas últimas, no es fácil encajar en esta categoría al prototipo de «leproso pobre». El mismo es válido para el adjetivo «excluido», especialmente si tenemos en cuenta los términos de comparación que recordaré a continuación.

Así, en dirección opuesta a los lázaros domésticos parecen estar los llamados «lázaros errantes por el mundo». Como el adjetivo indica, en esta situación se encontraban los enfermos que habían sido expulsados efectivamente de los núcleos urbanos donde residían y que no disponían de los medios para permanecer en su domicilio, ni oportunidad para ingresar en una leprosería. Por tanto, sólo les quedaba la dependencia de terceros y la errancia por el mundo en busca de limosnas en una situación que (ahora si) está marcada por la pobreza.

No nos debe asustar el hecho de que estos leprosos no tienen lugar en las leproserías. Lo mismo que sucedía en el resto del Occidente Medieval, las malaterías portuguesas funcionaban como instituciones locales en las que se impedía el acceso a los individuos que no tuviesen cualquier tipo de vínculo con el ayuntamiento al que la leprosería estaba asociada. Adicionando, que muchas ciudades tampoco disponían ni siquiera de una leprosería mientras en otras el acceso a la institución implicaba el pago de una comisión de entrada que no todos podrían costear.

Deambulando al margen de lo institucional, los lázaros errantes parecen, a primera vista, disolverse en una masa anónima e indiferenciada de enfermos que se mueve en un «lugar confuso donde [el leproso] iba a mezclar su lepra con la lepra de los otros» ${ }^{12}$. Al contrarios de algunos enfermos domésticos e de otros cuantos residentes en las leproserías, desconocemos por completo sus nombres o su proveniencia. Con todo, esta aparente oscuridad esconde otra percepción.

Todos los documentos en que se ha hecho mención a los lázaros errantes sugieren, por un lado, que la sociedad portuguesa de los siglos XIV y XV conocía perfectamente la situación de estos enfermos. Por otro, nos revelan mecanismos que acentuaban la diferenciación entre los enfermos «salvajes» y los restantes individuos leprosos, sobre todo con relación a los residentes en las leproserías.

Estas características están patentes, por ejemplo, en una legación testamentaria datada en 1367 que contempla a los leprosos de la malatería de Lisboa y los «de fuera de la

11 AHCML, Livro I do Hospital de São Lázaro, doc. 23.

12 FOUCAULT, Michel, op. cit., p. 88. 
malatería» ${ }^{13}$. Lo mismo es verificable en el ya referido conjunto de penas estipuladas por la edilidad de O Porto ${ }^{14}$ : si un residente en la malatería entrase clandestinamente en la ciudad debía ser expulsado del espacio urbano y le sería retirada la ración; si se tratase de un lázaro errante («que no tiene ración»), la misma infracción sería sólo castigada con la expulsión. No obstante, si el mismo lázaro errante volviera una segunda vez, además de la expulsión le esperaban represalias «con palos». A la tercera transgresión sería azotado públicamente por las calles de la ciudad y nuevamente expulsado.

Curiosamente, si comparamos estos dos testimonios - legación testamentaria y disposiciones punitivas - identificamos rápido uno de los trazos más reveladores de las actitudes de la sociedad medieval frente a sus miembros más desprovistos. Al mismo tiempo que aparecen preocupaciones caritativas, se demuestra ser capaz de actuar con una brutalidad impresionante en caso de que las reglas establecidas no sean cumplidas. Unas no anulan a la otra en un cuadro de aparentes paradojas y ambigüedades.

Pero no son estas las referencias más interesantes que identifiqué en el territorio portugués sobre los lázaros errantes por el mundo. En un carta redactada en 1365 ${ }^{15}$, D. Pedro I informa de la justicia de su reino que, oponiéndose a las ordenes que el y su padre habían estipulado antes de su reinado, a los leprosos «salvajes» se les impide pedir limosna en las grandes ciudades y en otros lugares del Reino. Como vimos, O Porto era ejemplo de eso. La diferencia es que, en el escenario relatado por el Rey, no era la justicia local que impedía a los leprosos pedir limosna, pero si los propios enfermos que residían en las leproserías los que, levantándose en conjunto con sus procuradores, agredían a los errantes.

Aquí creo que es adecuado retomar la caracterización que diseña Françoise Bériac sobre las comunidades de leprosos organizadas, refiriéndose a ellas como a una «caricatura de la sociedad sana» ${ }^{16}$. En este ejemplo vemos a los residentes de las leproserías protegiendo sus intereses, esforzándose por evitar la división de la caridad de los habitantes de la ciudad con los enfermos «de fuera», con los «extranjeros». Y también vemos otro aspecto digno de nota.

En realidad, nos damos cuenta de que los lázaros errantes no sólo se trasladaban a las ciudades en busca de limosnas. Buscaban igualmente una oportunidad para ingresar en las malaterías, esa busca contrariaba a los referidos intereses de los residentes y las determinaciones de las concejalías. De ahí que no los recibieran.

Más que no recibirlos, con todo, lo que me interesa son las motivaciones que están por detrás de la intención de entrar en la institución. Podemos adivinar que para estos enfermos la idea de recibir raciones diarias o mensuales fuera más llamativa que la dependencia de las limosnas. Independientemente de que existen otras posibles explicaciones, estas ocurrencias manifiestan el carácter relativo de la descripción de la leprosería como

13 IAN/TT, Mosteiro de Santo Agostinho, m. 2, n. ${ }^{\circ} 7$.

14 Vid. supra, nota 8.

15 Chancelarias Portuguesas - Chancelaria D. Pedro I (1357-1367), ed. MARQUES, A.H. Oliveira, Lisboa, INIC/Centro de Estudos Históricos da Universidade Nova de Lisboa, 1984, pp. 481-482.

16 BÉRIAC, Françoise: Histoire des Lépreux au Moyen Âge, une société d'exclus, Paris, Editions Imago, 1988 , p. 249. 
un espacio de «gran cierre» ${ }^{17}$ semejante a una prisión. Si esta pudo eventualmente haber sido la perspectiva de algunos leprosos, no parece haber sido compartida por el conjunto total de enfermos.

$\mathrm{Y}$ verificamos el mismo principio para el caso de los que efectivamente residieron en las leproserías.

Ya hemos tenido oportunidad de constatar que ni todos los que sufrían del «dolor de San Lázaro» eran recibidos en las leproserías urbanas, restringiéndose el acceso a aquellos que, como he afirmado, tenían vínculos con el espacio urbano donde la institución estaba localizada. No es absolutamente claro cual es el tipo de vínculo que posibilitaba el acceso, pero la mayoría de las ocurrencias parece apuntar a la naturalidad como factor determinante.

En cualquier caso, hasta nuestros días han llegado pruebas de una oscilación entre la hospitalización compulsiva y el internamiento voluntario. El estatuto de la Casa de San Lázaro de Lisboa, por ejemplo, apunta específicamente que todos los leprosos deben ser obligados a ingresar en la leprosería incluso «en contra de su voluntad» ${ }^{18}$. En un sentido inverso, otros enfermos deseaban un lugar en la institución pero, porque no eran naturales de la ciudad, allí no podían ser recibidos. Es lo que sucede con un enfermo que, en 1508, recibe autorización de la Reina para entrar en la leprosería de Lisboa ${ }^{19}$. Unos años después la misma autorización regia es tendida a la hija de Domingos Gonçalvez que, enfermando en una ciudad que no disponía de leprosería, procuró internarse voluntariamente en Lisboa ${ }^{20}$.

Fuese voluntaria o compulsiva, la entrada en la Casa señalaba una alteración importante en la vivencia cotidiana antes de la enfermedad. La obligatoriedad de obedecer a un conjunto de determinaciones (que en algunas instituciones de Occidente medieval eran semejantes o idénticas a las reglas monásticas) limitaba de una forma importante las opciones de los enfermos. Conforme a los períodos y a las instituciones, estos límites - que traducen claramente las principales preocupaciones de la sociedad sana en relación a los individuos enfermos - van sufriendo alteraciones, diseñándose en varios aspectos de la vida del residente: prácticas religiosas, relaciones matrimoniales e interpersonales, gestión de las herencias, contacto con el exterior.

A partir del momento en el que tenemos en cuenta que existieron diferentes grados de control ejercido sobre el leproso, percibimos que la institución no absorbe al individuo hasta el punto de declarar su «muerte civil». En realidad, el encuadramiento institucional no sólo impone deberes, también confiere un abanico de «derechos» o capacidades que varían de forma y de número (raciones, limosnas, representación jurídica). No siéndome posible analizar aquí todos los ejemplos disponibles, sólo haré referencia a un testimonio extraído de una carta de venta datada en el 1488:

17 FOUCAULT, Michel: Vigiar e Punir. Nascimento da Prisão, Petrópolis, Editora Vozes, 1987, p. 164.

18 «Regimento e estatuto feito sobre a Casa de S. Lázaro desta cidade de Lisboa» en Livro das Posturas Antigas, ed. RODRIGUES, Maria Teresa Campos, Lisboa, Câmara Municipal de Lisboa, 1974, p. 185.

19 AHCML, Livro I do Hospital de São Lázaro, doc. 18.

20 AHCML, Livro I do Hospital de São Lázaro, doc. 28. 
Con el objetivo de efectuar dicha venta de una propiedad que pertenecía a la Casa de San Lázaro de Lisboa, los lázaros se reúnen con el comprador en la leprosería donde discuten los términos de transacción. Recusando el valor acordado inicialmente, los residentes proponen otras condiciones y, terminada la discusión, reciben de inmediato parte de la cuantía establecida. Posteriormente, la venta es confirmada por el proveedor de la Casa que no estuvo presente en la celebración del contrato ${ }^{21}$.

El aspecto que sobresale primero es talvez el de la participación activa de los lázaros en la vida económica de la institución. Son ellos los que deciden el destino de la transacción sin tener la necesidad de consultar in loco al responsable de la leprosería. Aún, asistimos aquí a una ocasión de contacto entre el mundo sano y el de los enfermos que parece ser frecuente y perfectamente tolerado, al contrario de lo que pasaba, como vimos, con los lázaros errantes por el mundo. Y que distinta era la situación en la que estos se encontraban...

\section{CONCLUSIONES: EL LEPROSO ADEMÁS DE LA LEPRA}

Dada la riqueza da documentación que hasta aquí he ido presentando, muchos otros enfoques se podrían realizar desde una mirada mas demorada y otros tantos comentarios merecerían ser evocados en este trabajo. Con todo - tempus fugit- he optado por enfocar la atención del lector en un aspecto que creo que se sobrepone a todos los otros: la búsqueda del leproso además de la lepra.

Así, a propósito me he desviado de las percepciones medievales de aquella enfermedad tan específica, de las actitudes sociales frente a la enfermedad y al enfermo, de las características institucionales de las leproserías. Por detrás de estos elementos y sin dejar de tenerlos en consideración, he procurado centrar la reflexión historiográfica en el propio enfermo y atribuirle el papel de conductor del análisis.

Este ha sido el esquema que he considerado más eficaz para oponerme a la tendencia de encarar a los leprosos como una categoría «especial» de la sociedad medieval, rápidamente etiquetada de «marginal» porque «enferma». Ha escrito Jérôme Baschet que «...il serait fort difficile, au Moyen Âge, de concevoir la personne indépendamment des groupes et des communautés au sein desquels elle vit» ${ }^{22}$. Creo que el mismo principio es aplicable en el caso de los hombres y mujeres que han sufrido de lepra. Independientemente del grado de revisión de los papeles sociales y de las vivencias cotidianas anteriores a la enfermedad, los aspectos que definían al individuo son los que determinan la transición para la condición del enfermo y permanecen válidos en el período de la enfermedad.

21 AHCML, Livro I do Hospital de São Lázaro, doc. 14.

22 BASCHET, Jérôme: La civilisation féodale de l'an mil à la colonisation de l'Amérique, Paris, Éditions Flammarion, 2006, p. 633. 


\section{BIBLIOGRAFÍA}

ARRIZABALAGA, Jon: «La identificación de las causas de muerte en la Europa preindustrial: algunas consideraciones historiográficas», Boletín de la Asociación de Demografía Histórica, 11-3 (1993), pp. 23-47.

ARRIZABALAGA, Jon: «Problematizing retrospective diagnosis in the history of the disease», Asclepio, LIV-1 (2002), pp. 51-70.

AUGÉ, Marc: «Biological order, social order: illness, a primary form of event», in The Meaning of Illness (ed. AUGÉ, Marc; HERZLICH, Claudine), [s.l.], Harwood Academic Publishers, 1995, pp. 23-70.

BASCHET, Jérôme: La civilisation féodale de l'an mil à la colonisation de l'Amérique, Paris, Éditions Flammarion, 2006.

BÉRIAC, Françoise: Histoire des Lépreux au Moyen Âge, une société d'exclus, Paris, Editions Imago, 1988.

CUNNINGHAM, Andrew: «Identifying disease in the past: cutting the Gordian knot», Asclepio, LIV-1 (2002), pp. 13-34.

FOUCAULT, Michel: «O nascimento da medicina social», in A microfísica do poder, Brasil, Edições Graal, 2008, pp. 79-98.

FOUCAULT, Michel: Vigiar e Punir. Nascimento da Prisão, Petrópolis, Editora Vozes, 1987.

GEARY, Patrick: $O$ Mito das Nações. A invenção do nacionalismo, Lisboa, Gradiva, 2008.

RAWCLIFFE, Carole: Leprosy in Medieval England, Woodbridge, The Boydell Press, 2006.

ROSENBERG, Charles E.: «Introduction. Framing Disease: Illness, Society, and History», in Framing Disease: studies in cultural history (ed. ROSENBERG, Charles E.; GOLDEN, Jane), EUA, Rutgers, 1997, pp. XIII-XXVI.

TOUATI, François-Olivier: «Contagion and leprosy: myth, ideas and evolution in medieval minds and societies», in Contagion: perspectives from Pre-modern society, Ashgate, 2000, pp. 179-201.

TOUATI, François-Olivier: Maladie et société au Moyen Âge. La lèpre, les lépreux et les léproseries dans la province ecclésiastique de Sens jusqu'au milieu du XIVe siècle, Paris, De Boeck Université, 1998.

TOUATI, François-Olivier: «Un dossier à rouvrir: l'assistance au Moyen Âge», in Fondations et œuvres charitables au Moyen Âge. Actes du 121e congrès national des sociétés historiques et scientifiques, Paris, C.T.H.S., 1999, pp. 23-38. 